\title{
UNCERTAINTY QUANTIFICATION IN NONLINEAR STRUCTURAL DYNAMICS FOR MISTUNED BLADED DISKS.
}

\author{
Evangéline Capiez-Lernout $^{1}$, Christian Soize ${ }^{1}$, and Moustapha Mbaye ${ }^{2}$ \\ ${ }^{1}$ Université Paris-Est, Laboratoire Modélisation et Simulation Multi-chelle, MSME UMR 8208 CNRS \\ 5 bd Descartes, 77454 Marne-la-Vallée Cedex 02 \\ e-mail: \{evangeline.capiez-lernout,christian.soize\}@u-pem.fr \\ 2 Turbomeca - Safran Group \\ 64511 Bordes, France \\ e-mail: moustapha.mbaye@safran.fr
}

Keywords: Nonlinear mistuning, Uncertainty quantification, Geometrical nonlinearities, Bladed disks, Reduced-order model.

\begin{abstract}
The recent improvements in turbomachinery design combined to the unavoidable requirements of kerosene savings require to analyze the exceptional operating regime of bladed disks, for which large deformations and large displacements can occur. It seems then quite appropriate to consider the geometrically nonlinear effects in the computational models dedicated to the analysis of mistuned turbomachinery bladed-disks. A special attention has to be first given to the case of geometrically nonlinear tuned bladed disks. The large set of nonlinear coupled differential equations issued from the finite element model of the tuned structure has to necessarily be solved in the time domain, leading us to establish a reduced-order strategy. The operators of the corresponding mean nonlinear reduced-order model are then deduced from its explicit construction as shown in the context of three-dimensional solid finite elements. One also has to focus on the modeling of the external load, corresponding to a frequency band chosen for the excitation, which has to be selected according to usual turbomachinery criterions. The external load has to be defined in the time domain but has to represent a uniform sweep in the frequency domain. We then propose to implement the mistuning uncertainties by using the nonparametric probabilistic framework. We then obtain a stochastic reduced-order model, which requires to solve a reasonable set of uncertain nonlinear coupled differential equations in the time domain, yielding appropriate efficient and dedicated algorithms to be constructed. Such computational strategy provides an efficient computational tool, which is applied on a finite element model of an industrial centrifugal compressor with a large number of degrees of freedom. This allows new high complex dynamical behaviors to be put in evidence.
\end{abstract}




\section{INTRODUCTION}

In general, the natural cyclic symmetry of turbomachinery bladed disks is broken because of manufacturing tolerances and material dispersions, which create small variations from one blade to another one. Such phenomena, referred to mistuning, can generate localization effects combined to a dynamic amplification of the forced response [1]. Many research efforts have been carried out on this subject, including reduced-order models with probabilistic approaches in the numerical modeling, for taking into account the random character of mistuning $[2,3]$ and giving rise to strategies for the robust design of such structures [4]. Another essential aspect is to consider the geometric nonlinear effects in the computational models occurring when exceptional operating speeds of bladed disks are analyzed due to geometric nonlinearities induced by large deformations and large displacements $[5,6]$. Such situation is realistic when considering a flutter kind phenomenon induced by unsteady aerodynamic coupling and yielding low damping levels. Since the unsteady aerodynamic coupling is not considered in this paper, the nonlinear domain is simulated by increasing the magnitude of the external load, while performing forced response calculations. The present paper proposes a methodology adapted to geometric nonlinear analysis of mistuned bladed disks combined with an industrial realistic application. The first part is devoted to the development of an adapted nonlinear reduced-order computational model for the tuned structure, referred as the MEAN-NL-ROM. It is explicitly constructed in the context of three-dimensional solid finite elements [7] by using an appropriate projection basis [8] obtained here by a linear tuned eigenvalue analysis. In a second Section, once the MEAN-NL$\mathrm{ROM}$ is established, mistuning is taken into account by implementing uncertainties through the nonparametric probabilistic framework $[9,10]$, yielding a nonlinear stochastic computational model referred as the STOCH-NL-ROM. The numerical algorithm used for solving such set of nonlinear stochastic coupled differential equations is explained. Finally, a numerical application, consisting of a finite-element model of an industrial integrated bladed disk with about 2 , 000,000 dof is considered. The geometric nonlinear effects are analyzed and quantified through the dynamic analysis of the magnification factor in both tuned and mistuned cases.

\section{CONSTRUCTION OF THE MEAN-NL-ROM RELATED TO THE TUNED STRUC- TURE}

This Section is devoted to the construction of the MEAN-NL-ROM related to rotating bladeddisks structures with cyclic symmetry. In the present work, the bladed disk under consideration is assumed (1) to be made up of a linear elastic material and (2) to undergo large displacements and large deformations inducing geometrical nonlinearities.

\subsection{Description of the geometric nonlinear boundary value problem}

The structure under consideration is a bladed-disk structure with a $M$-order cyclic symmetry. Thus, the geometrical domain, the material constitutive equation, and the boundary conditions related to the generating sector are invariant under the $2 \pi / M$ rotation around its axis of symmetry. Moreover, the bladed disk undergoes a rotational motion around the axis of symmetry with constant angular speed $\Omega$. The structure is made up of a linear elastic material and is assumed to undergo large deformations inducing geometrical nonlinearities. A total Lagrangian formulation is chosen, which means that the dynamical equations are expressed in the rotating frame of an equilibrium configuration considered as a prestressed static configuration. Let $\Omega$ be the three-dimensional bounded domain corresponding to such reference configuration and subjected to the body force field $\mathbf{g}(\mathbf{x}, t)$, in which $\mathbf{x}$ denotes the position of a given point belonging 
to domain $\boldsymbol{\Omega}$. The boundary $\partial \boldsymbol{\Omega}$ is such that $\partial \boldsymbol{\Omega}=\boldsymbol{\Gamma} \cup \boldsymbol{\Sigma}$ with $\boldsymbol{\Gamma} \cap \boldsymbol{\Sigma}=\emptyset$ and the external unit normal to boundary $\partial \boldsymbol{\Omega}$ is denoted by $\mathbf{n}$. The boundary part $\boldsymbol{\Gamma}$ corresponds to the fixed part of the structure (in the local rotating frame $\left(\mathbf{x}_{1}, \mathbf{x}_{2}, \mathbf{x}_{3}\right)$ ) whereas the boundary part $\boldsymbol{\Sigma}$ is subjected to the external surface force field $\mathbf{G}(\mathbf{x}, t)$. Note that the external force fields are derived from the Lagrangian transport into the reference configuration of the physical body/surface force fields applied in the deformed configuration. The external load can represent, for instance, the unsteady pressures applied to the blades. The displacement field expressed with respect to the reference configuration is denoted as $\mathbf{u}(\mathbf{x}, t)$.

\subsection{MEAN-NL-ROM}

Let $\mathcal{C}$ be the admissible space defined by

$$
\mathcal{C}=\{\mathbf{v} \in \Omega, \mathbf{v} \text { sufficiently regular }, \mathbf{v}=\mathbf{0} \text { on } \Gamma\} .
$$

The vector $\mathbf{q}=\left(q_{1}, \ldots, q_{N}\right)$ of the generalized coordinates is then introduced as a new set of unknown variables by projecting the reference nonlinear response $\mathbf{u}(\mathbf{x}, \cdot)$ on the vector space spanned by the finite family $\left\{\varphi^{1}, \cdots, \varphi^{N}\right\}$ of a given vector basis of $\mathcal{C}$. The MEAN-NL-ROM is then described by the approximation $\mathbf{u}^{N}(\mathbf{x}, t)$ of order $N$ of $\mathbf{u}(\mathbf{x}, t)$ such that

$$
\mathbf{u}^{N}(\mathbf{x}, t)=\sum_{\beta=1}^{N} \varphi^{\beta}(\mathbf{x}) q_{\beta}(t),
$$

in which $\mathbf{q}$ is solution of the nonlinear differential equation

$$
\mathcal{M}_{\alpha \beta} \ddot{q}_{\beta}+\left(\mathcal{D}_{\alpha \beta}+\mathcal{C}(\Omega)_{\alpha \beta}\right) \dot{q}_{\beta}+\left(\mathcal{K}_{\alpha \beta}^{(e)}+\mathcal{K}^{(c)}(\Omega)_{\alpha \beta}+\mathcal{K}_{\alpha \beta}^{(g)}\right) q_{\beta}+\mathcal{K}_{\alpha \beta \gamma}^{(2)} q_{\beta} q_{\gamma}+\mathcal{K}_{\alpha \beta \gamma \delta}^{(3)} q_{\beta} q_{\gamma} q_{\delta}=\mathcal{F}_{\alpha} .
$$

The usual convention of summation over Greek or Latin repeated indices is used. Let $\rho(\mathbf{x})$ be the mass density field expressed in the reference configuration and let $a=\left\{a_{i j k \ell}\right\}_{i j k \ell}$ be the fourth-order elasticity tensor, which satisfies the usual symmetry, boundedness, and positivedefiniteness properties. In Eq. (3), the reduced operators $[\mathcal{M}],\left[\mathcal{K}^{(g)}\right]$ and $\left[\mathcal{K}^{(e)}\right]$ are the mass, geometrical stiffness, and elastic stiffness real $(N \times N)$ matrices with positive-definiteness property, for which entries are defined by

$$
\begin{gathered}
\mathcal{M}_{\alpha \beta}=\int_{\boldsymbol{\Omega}} \rho \varphi_{i}^{\alpha} \varphi_{i}^{\beta} d \mathbf{x}, \\
\mathcal{K}_{\alpha \beta}^{(e)}=\int_{\boldsymbol{\Omega}} a_{j k \ell m} \varphi_{j, k}^{\alpha} \varphi_{\ell, m}^{\beta} d \mathbf{x}, \\
\mathcal{K}_{\alpha \beta}^{(g)}=\int_{\boldsymbol{\Omega}} \sigma_{i j}^{(g)} \varphi_{s, i}^{\alpha} \varphi_{s, j}^{\beta} d \mathbf{x},
\end{gathered}
$$

where the second-order symmetric tensor field $\sigma^{(g)}=\left\{\sigma_{i j}^{(g)}\right\}_{i j}$ represents the Cauchy constraints acting on the reference configuration, and where $\psi_{i, j}=\frac{\partial \psi_{i}}{\partial x_{j}}$. The rotational effects are taken into account through the reduced operators $[\mathcal{C}(\Omega)]$ and $\left[\mathcal{K}^{(c)}(\Omega)\right]$, which represent the gyroscopic coupling term with antisymmetry property and the centrifugal stiffness term with negative-definiteness property, which are written as

$$
\mathcal{C}_{\alpha \beta}(\Omega)=\int_{\boldsymbol{\Omega}} 2 \rho[R]_{i j} \varphi_{j}^{\alpha} \varphi_{i}^{\beta} d \mathbf{x}
$$




$$
\mathcal{K}_{\alpha \beta}^{(c)}(\Omega)=\int_{\boldsymbol{\Omega}} \rho[R]_{i k}[R]_{k j} \varphi_{j}^{\alpha} \varphi_{i}^{\beta} d \mathbf{x}
$$

where the $(3 \times 3)$ matrix $[R]$ is such that $[R]_{i j}=-\Omega \varepsilon_{i j 3}$, where $\varepsilon_{i j k}$ is the Levi-Civita symbol such that $\varepsilon_{i j k}= \pm 1$ for an even or odd permutation and $\varepsilon_{i j k}=0$ otherwise. It should be noted that the centrifugal effects are assumed to be sufficiently small so that the linear stiffness reduced matrix $[\mathcal{K}(\Omega)]=\left[\mathcal{K}^{(e)}\right]+\left[\mathcal{K}^{(c)}(\Omega)\right]+\left[\mathcal{K}^{(g)}\right]$ is positive definite, yielding only stable dynamical systems to be considered. The geometric nonlinearities are taken into account through the quadratic and cubic stiffness contributions $\mathcal{K}_{\alpha \beta \gamma}^{(2)}$ and $\mathcal{K}_{\alpha \beta \gamma \delta}^{(3)}$ which are written as

$$
\begin{gathered}
\mathcal{K}_{\alpha \beta \gamma}^{(2)}=\frac{1}{2}\left(\widehat{\mathcal{K}}_{\alpha \beta \gamma}^{(2)}+\widehat{\mathcal{K}}_{\beta \gamma \alpha}^{(2)}+\widehat{\mathcal{K}}_{\gamma \alpha \beta}^{(2)}\right), \\
\widehat{\mathcal{K}}_{\alpha \beta \gamma}^{(2)}=\int_{\boldsymbol{\Omega}} a_{j k \ell m} \varphi_{j, k}^{\alpha} \varphi_{s, \ell}^{\beta} \varphi_{s, m}^{\gamma} d \mathbf{x}, \\
\mathcal{K}_{\alpha \beta \gamma \delta}^{(3)}=\frac{1}{2} \int_{\boldsymbol{\Omega}} a_{j k \ell m} \varphi_{r, j}^{\alpha} \varphi_{r, k}^{\beta} \varphi_{s, \ell}^{\gamma} \varphi_{s, m}^{\delta} d \mathbf{x} .
\end{gathered}
$$

It can easily be shown that tensor $\mathcal{K}_{\alpha \beta \gamma}^{(2)}$ has permutation-invariance property and that tensor $\mathcal{K}_{\alpha \beta \gamma \delta}^{(3)}$ has positive-definiteness property. Concerning the reduced damping operator, a modal damping model is added. Finally, the reduced external load is written as

$$
\mathcal{F}_{\alpha}=\int_{\boldsymbol{\Omega}} g_{i} \varphi_{i}^{\alpha} d \mathbf{x}+\int_{\Sigma} G_{i} \varphi_{i}^{\alpha} d \mathbf{s}
$$

Concerning the choice of the projection basis, the one related to the linear eigenvalue problem of the rotating tuned conservative structure, for which the gyroscopic coupling effects are ignored, is chosen. Since the tuned structure has a perfect cyclic symmetry, the use of the discrete Fourier transform allows for rewriting the eigenvalue problem of the whole tuned structure into uncoupled sub-eigenvalue problems related to the generator sector with appropriate boundary conditions $[13,14]$. The eigenvectors of the whole tuned structure are then reconstructed in its corresponding physical space. Note that these eigenvectors are ordered by increasing values of their corresponding eigenvalues $\lambda_{\alpha}, \alpha \in\{1, \ldots, N\}$ and verify the following orthogonality properties,

$$
\mathcal{M}_{\alpha \beta}=\delta_{\alpha \beta} \quad, \quad \mathcal{K}_{\alpha \beta}^{(g)}+\mathcal{K}^{(c)}(\Omega)_{\alpha \beta}+\mathcal{K}_{\alpha \beta}^{(e)}=\lambda_{\alpha} \delta_{\alpha \beta},
$$

where $\delta_{\alpha \beta}$ is the Kronecker symbol such that $\delta_{\alpha \beta}=1$ if $\alpha=\beta$ and $\delta_{\alpha \beta}=0$ otherwise. It should be noted that such projection basis issued from a linear eigenvalue problem is used for the construction of the MEAN-NL-ROM, which means that a systematic convergence analysis with respect to $N$ is carried out so that the MEAN-NL-ROM is representative of the nonlinear dynamical behavior of the structure.

\subsection{Numerical aspects for the construction of the nonlinear reduced operators}

The MEAN-NL-ROM is explicitly constructed from the knowledge of the projection basis. It is carried out in the context of the three-dimensional finite element method, for which the finite elements are chosen as isoparametric solid finite elements with 8 nodes with a numerical integration using 8 Gauss integration points. Concerning the construction of the quadratic and 
cubic nonlinear stiffness operators, the methodology presented in [11] is used. In particular, due to the chosen strategy for the mistuning modeling, it should be underlined that the $\widehat{\mathcal{K}}_{\alpha \beta \gamma}^{(2)}$ and $\mathcal{K}_{\alpha \beta \gamma \delta}^{(3)}$ nonlinear stiffness entries have to be explicitly known. The numerical procedure uses the symmetry properties of the reduced operators, combined with the use of distributed calculations in parallel computer in order to optimize its efficiency. The main steps, which can be found in details in [11], require (1) the computation of the elementary contributions of each type of internal forces projected on the vector basis, (2) the finite element assembly of these elementary contributions, (3) the computation of the reduced operators by projecting each assembled internal force on the projection vector basis.

\subsection{Strategy for the construction of the external load}

In the present case, the presence of the geometric nonlinearities yields the nonlinear differential equation Eq. (3) to be solved in the time domain, the frequency content of the nonlinear dynamical response being a posteriori post-analyzed by using a Fast-Fourier-Transform (FFT). The reduced excitation issued from the external load is assumed to be splitted according to a spatial part and to a time-domain part such that

$$
\mathcal{F}_{\alpha}(t)=f_{0} r_{\alpha} g(t)
$$

in which $f_{0}$ is a coefficient characterizing the global load intensity, where $\mathbf{r}$ is a $\mathbb{R}^{N}$-vector corresponding to the spatial modal contribution of the external load, and where $g(t)$ describes the time evolution of the load. Similarly to the usual linear analysis of structures with $M$-order cyclic symmetry, the excitation is constructed with a cyclic spatial repartition and a constant phase shift $(2 \pi h) / M$ from one blade to another one, so that only the eigenfrequencies corresponding to a given $h$ circumferential wave number are excited. Note that the use of the cyclic symmetry property has no real interest for expressing the nonlinear response according to its harmonic components because a decoupling between the harmonic components cannot be obtained. Moreover, it should be recalled that the usual linear analysis of bladed-disk structures requires to display the eigenfrequencies of the structure with respect to its circumferential wave number. It can be shown that the eigenmodes corresponding to localized blade modes are characterized by straight lines contrary to the eigenmodes corresponding to global coupled diskblade modes. It is well known that the mistuning important effects of response amplification are concentrated in "veering" zones for which the coupling between the disk motion and the blade motion is the strongest. For this reason, the nonlinear dynamical analysis has to be performed in a chosen frequency band of excitation and not for a single frequency excitation. Because of the geometric nonlinearities, the use of a harmonic excitation seems to be inappropriate because the set of nonlinear coupled differential equations should be solved for each harmonic excitation considered. The strategy is to simultaneously and uniformly excite all the frequencies of the given frequency band of excitation so that only one computation of the nonlinear dynamical problem is required. In Eq. (14), the function $g(t)$ is defined by

$$
g(t)=\frac{2 \pi \Delta \nu}{\pi} \operatorname{sinc}_{\pi}(t \Delta \nu) \cos (2 \pi s \Delta \nu t)
$$

where $x \mapsto \operatorname{sinc}_{\pi}(x)$ is the function defined by $\operatorname{sinc}_{\pi}(x)=\sin (\pi x) /(\pi x)$. Note that the Fourier transform of such function is

$$
\widehat{g}(2 \pi \nu)=\mathbb{1}_{\widetilde{\mathbb{B}}_{e}}(2 \pi \nu)
$$


in which $\mathbb{1}_{\widetilde{B}}(x)=1$ if $x \in \mathbb{B}$ and 0 otherwise, and where $\widetilde{\mathbb{B}}_{e}=\left\{-\mathbb{B}_{e}\right\} \bigcup\left\{\mathbb{B}_{e}\right\}$ with

$$
\mathbb{B}_{e}=[2 \pi(s-1 / 2) \Delta \nu, 2 \pi(s+1 / 2) \Delta \nu] .
$$

It should be noted that such time-evolution excitation allows a forced-response problem and not a time-evolution problem with initial conditions to be considered. The forced-response problem is thus approximated by an equivalent time-evolution problem with zero initial conditions over a finite time interval, which includes almost all of the signal energy of the excitation.

\section{UNCERTAINTY QUANTIFICATION INDUCED BY THE MISTUNING}

The random nature of the mistuning is then considered by implementing the nonparametric probabilistic approach, which presents the capability to include both the system-parameter uncertainties and the model uncertainties induced by modeling errors (see [9] for a complete review on the subject). Since the analysis is carried out for the class of integrated bladed disks that are manufactured from a unique solid piece of material, the uncertainties are not considered to be independent from one blade to another one (in opposite to the case of a fan). The MEAN-NL-ROM is constructed by modal analysis without any sub-structuring techniques.

\subsection{Nonparametric probabilistic model for the mistuning}

It is assumed that only the linear operators of the structure are concerned with the mistuning phenomenon. The linear reduced operators $[\mathcal{M}],[\mathcal{D}],[\mathcal{C}(\Omega)],\left[\mathcal{K}^{(g)}\right],\left[\mathcal{K}^{(c)}(\Omega)\right]$, and $\left[\mathcal{K}^{(e)}\right]$ of the MEAN-NL-ROM are replaced by the random matrices $[\mathcal{M}],[\mathcal{D}],[\mathcal{C}(\Omega)],\left[\mathcal{K}^{(g)}\right],\left[\mathcal{K}^{(c)}(\Omega)\right]$, and $\left[\mathcal{K}^{(e)}\right]$ defined on the probability space $(\Theta, \mathcal{T}, \mathcal{P})$ such that $\mathcal{E}\{[\mathcal{M}]\}=[\mathcal{M}], \mathcal{E}\{[\mathcal{D}]\}=[\mathcal{D}]$, $\mathcal{E}\{[\mathcal{C}(\Omega)]\}=[\mathcal{C}(\Omega)], \mathcal{E}\left\{\left[\mathcal{K}^{(g)}\right]\right\}=\left[\mathcal{K}^{(g)}\right], \mathcal{E}\left\{\left[\mathcal{K}^{(c)}(\Omega)\right]\right\}=\left[\mathcal{K}^{(c)}(\Omega)\right]$, and $\mathcal{E}\left\{\left[\mathcal{K}^{(e)}\right]\right\}=\left[\mathcal{K}^{(e)}\right]$, in which $\mathcal{E}$ is the mathematical expectation.

Let $[\mathcal{A}]$ be a $(N \times N)$ matrix issued from the MEAN-NL-ROM with positive-definite property. For instance, it represents, the mass, the damping, the geometrical stiffness, the linear elastic stiffness or the centripetal stiffness. The corresponding random matrix $[\mathcal{A}]$ is then written as

$$
[\mathcal{A}]=\left[L_{A}\right]^{T}\left[\mathbf{G}_{A}\left(\delta_{A}\right)\right]\left[L_{A}\right]
$$

in which $\left[L_{A}\right]$ is the $(N \times N)$ upper triangular matrix issued from the Cholesky factorization of $[\mathcal{A}]$, and where $\left[\mathbf{G}_{A}\right]$ is a full random matrix with values in the set of all the positive-definite symmetric $(N \times N)$ matrices.

When $[\mathcal{A}]$ is the gyroscopic coupling matrix, the corresponding random matrix $[\mathcal{A}]$ is then written as

$$
[\mathcal{A}]=\left[U_{A}\right]\left[L_{A}\right]^{T}\left[\mathbf{G}_{A}\left(\delta_{A}\right)\right]\left[L_{A}\right],
$$

in which the matrices $\left[U_{A}\right]$ and $\left[L_{A}\right]$ are the $(N \times N)$ matrices defined by $\left[L_{A}\right]=\left[S_{A}\right]^{1 / 2}\left[B_{A}\right]^{T}$ and $\left[U_{A}\right]=[\mathcal{A}]\left[B_{A}\right]\left[S_{A}\right]\left[B_{A}\right]^{T}$, in which the $(N \times N)$ full matrix $\left[B_{A}\right]$ is constituted of the eigenvectors of $[\mathcal{A}][\mathcal{A}]^{T}$ and where the $(N \times N)$ diagonal matrix $\left[S_{A}\right]$ is the singular values, issued from the single value decomposition (SVD) of matrix $[\mathcal{A}]$. Note that the probability distribution and the random generator of $\left[\mathbf{G}_{A}\left(\delta_{A}\right)\right]$ is detailed in [9]. The dispersion of random matrix $\left[\mathbf{G}_{A}\right]$ is controlled by the hyperparameter $\delta_{A}$ belonging an admissible set $\mathcal{D}$. Consequently, the mistuning level of the bladed-disk is entirely controlled by the $\mathbb{R}^{6}$-valued hyperparameter $\delta=\left(\delta_{M}, \delta_{D}, \delta_{C}, \delta_{K_{g}}, \delta_{K_{c}}, \delta_{K}\right)$, belonging to the admissible set $\mathcal{D}^{6}$. 


\subsection{STOCH-NL-ROM of the mistuned bladed-disk}

For constructing the STOCH-NL-ROM, the deterministic matrices in Eq. (3) are replaced by the random matrices. The deterministic displacement field $\mathbf{u}^{N}(\cdot, t)$ becomes a random field $\mathbf{U}^{N}(\cdot, t)$ that is written, for all $\mathbf{x} \in \boldsymbol{\Omega}$, as

$$
\mathbf{U}^{N}(\mathbf{x}, t)=\sum_{\beta=1}^{N} \varphi^{\beta}(\mathbf{x}) \mathbf{Q}_{\beta}(t),
$$

in which the $\mathbb{R}^{N}$-valued random variable $\mathbf{Q}(t)=\left(\mathbf{Q}_{1}(t), \cdots, \mathbf{Q}_{N}(t)\right)$ is solution of the following set of stochastic nonlinear differential equations,

$\mathcal{M}_{\alpha \beta} \ddot{\mathbf{Q}}_{\beta}+\left(\mathcal{D}_{\alpha \beta}+\mathcal{C}(\Omega)_{\alpha \beta}\right) \dot{\mathbf{Q}}_{\beta}+\left(\mathcal{K}_{\alpha \beta}^{(e)}+\mathcal{K}^{(c)}(\Omega)_{\alpha \beta}+\mathcal{K}_{\alpha \beta}^{(g)}\right) \mathbf{Q}_{\beta}+\mathcal{K}_{\alpha \beta \gamma}^{(2)} \mathbf{Q}_{\beta} \mathbf{Q}_{\gamma}+\mathcal{K}_{\alpha \beta \gamma \delta}^{(3)} \mathbf{Q}_{\beta} \mathbf{Q}_{\gamma} \mathbf{Q}_{\delta}=\mathcal{F}_{\alpha}$

\subsection{Numerical aspects for solving the STOCH-NL-ROM}

The solution of the STOCH-NL-ROM is calculated using the Monte Carlo numerical simulation. For each realization $\theta$ belonging to $\Theta$, the set of $N$ deterministic nonlinear coupled differential equations is considered and solved with an implicit and unconditionally stable integration scheme (Newmark method with the averaging acceleration scheme). Introducing the notation $\mathbf{Q}_{i}(\theta)=\mathbf{Q}\left(t_{i} ; \theta\right)$, related to each sample time $t_{i}$, the following set of $N$ deterministic nonlinear equations is solved for computing $\mathbf{Q}_{i}(\theta)$

$$
\left[\mathcal{K}_{i}^{\text {eff }}(\theta)\right] \mathbf{Q}_{i}(\theta)+\mathbf{F}^{\mathrm{NL}}\left(\mathbf{Q}_{i}(\theta)\right)=\mathcal{F}_{i}^{\text {eff }}(\theta),
$$

in which the effective $(N \times N)$ matrix $\left[\mathcal{K}_{i}^{\text {eff }}(\theta)\right]$ and the effective force vector $\mathcal{F}_{i}^{\text {eff }}(\theta)$ are easily computed at each time $t_{i}$. Note that matrix $\left[\mathcal{K}_{i}^{\text {eff }}(\theta)\right]$ has no particular signature due to the presence of the gyroscopic coupling matrix. The nonlinear term $\mathbf{F}^{\mathrm{NL}}\left(\mathbf{Q}_{i}(\theta)\right)$, issued from the presence of the geometric nonlinearities, is written as

$$
F_{\alpha}^{\mathrm{NL}}\left(\mathbf{Q}_{i}(\theta)\right)=\mathcal{K}_{\alpha \beta \gamma}^{(2)} Q_{i, \beta}(\theta) Q_{i, \gamma}(\theta)+\mathcal{K}_{\alpha \beta \gamma \delta}^{(3)} Q_{i, \beta}(\theta) Q_{i, \gamma}(\theta) Q_{i, \delta}(\theta)
$$

For each sampling time $t_{i}$, the computation of solution $\mathbf{Q}_{i}(\theta)$ is addressed by the fixed point method because this iterative scheme is few time consuming and does not require the evaluation of the tangential matrix. Nevertheless, when the algorithm does not converge, it is replaced by the Crisfield arc-length method [?]. Such algorithm introduces a new additional scalar parameter $u_{i}$ that multiplies the right-hand side member of the nonlinear equation. In this case, at each sampling time $t_{i}$, this nonlinear equation is written as

$$
\left[\mathcal{K}_{i}^{\text {eff }}(\theta)\right] \mathbf{Q}_{i}(\theta)+\mathbf{F}^{\mathrm{NL}}\left(\mathbf{Q}_{i}(\theta)\right)=u_{i}(\theta) \mathcal{F}_{i}^{\text {eff }}(\theta) .
$$

The nonlinear equation is solved step by step, each incremental step being characterized by a given arc length. For a given step, an iterative scheme requiring one evaluation of the tangential matrix allows a solution $\left(\mathbf{Q}_{i}(\theta), \Perp_{i}(\theta)\right)$ to be computed. Note that the tangential matrix can algebraically evaluated from Eq. (23). An adaptive arc length, depending on the number of iterations necessary to obtain the convergence of the preceding increment is also implemented according to [?] in order to accelerate the computation. An unusual procedure is then added to the algorithm, because Eq. (22) has to be solved instead of Eq. (24). In the nonlinear dynamical 
context, parameter $\mu_{i}$ is deterministic and has to be controlled in order to reach the value 1 . This implies that the state of the algorithm corresponding to the preceding increment has to be stored. When $u_{i}(\theta)$ is found to be upper than 1 , the algorithm is rewind to the preceding increment and the computation is set again with the half of the arc-length. Such additional procedure is repeated until parameter $\mu_{i}(\theta)$ reaches 1 within a numerical tolerance set to $10^{-6}$. Even if such procedure is time consuming, because of the necessary evaluations of the tangential matrix and due to the procedure controlling the value of parameter $\mu_{i}$, its main advantage is its capability of capturing high-nonlinear mechanical behaviors.

\section{NUMERICAL APPLICATION}

The structure under consideration in an industrial centrifugal compressor belonging to the class of integrated bladed disks. Due to proprietary reasons, the number $M$ of blades characterizing the order of the cyclic symmetry of the structure cannot given. The finite element model of the structure is constructed with solid finite elements and is constituted of about 2,000,000 degrees of freedom. The structure is in rotation around its revolution axis with a constant velocity $\Omega=30,750 \mathrm{rpm}$. Since the dynamic analysis is carried out in the rotating frame of the structure, the rigid body motion due to the rotation of the structure corresponds to a fixed boundary condition at the inner radius of the structure. The bladed disk is made up of a homogeneous isotropic material. A modal damping model is added for the bladed disk.

The cyclic symmetry is used for constructing the reduced matrices of the mean linear reducedorder model (MEAN-L-ROM). Let $\nu_{0}$ be the first eigenfrequency. In the application, a $5-t h$ engine-order excitation located at the tip of each blade is considered. The excitation frequency band is chosen such that $\mathbb{B}_{e}^{2}=[1.78,2.34]$ and is characterized by function $g(t)$ defined with parameters $s=4, \frac{\Delta \nu}{\nu_{0}}=0.51$ from the initial time $\nu_{0} t_{i n i}=-11.79$ and with a time duration $\nu_{0} T=184$. Figure 1 shows the graphs $t / t_{0} \mapsto g(t)$ and $\nu / \nu_{0} \mapsto \widehat{g}(2 \pi \nu)$ for excitation frequency band $\mathbb{B}_{e}^{2}$.
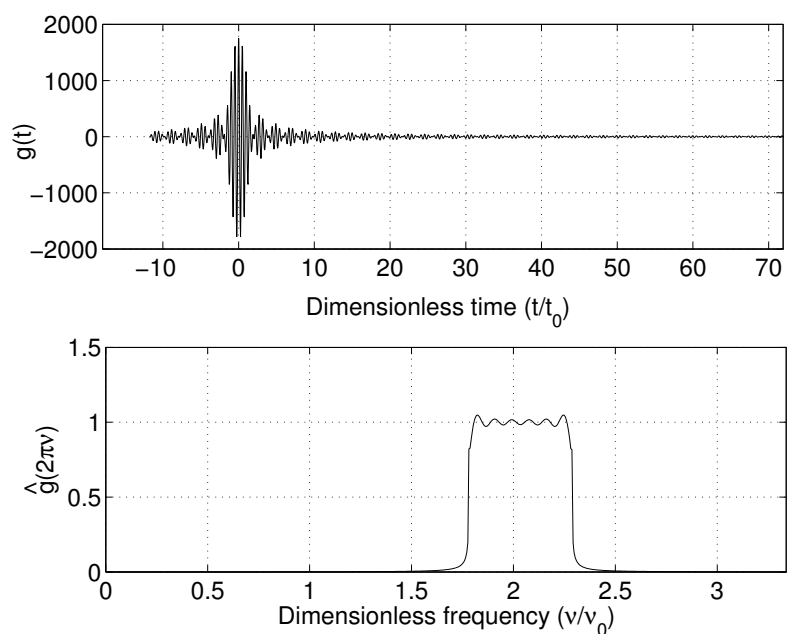

Figure 1: Representation of the external load in the time domain and in the frequency domain: graph of $t / t_{0} \mapsto g(t)$ (upper graph) and $\nu / \nu_{0} \mapsto \widehat{g}(2 \pi \nu)$ (lower graph) for $\mathbb{B}_{e}^{2}=[1.78,2.34]$.

The chosen observation is the displacement $u_{j}(t)$ corresponding to the out-plane displacement located at the tip of each blade $j$. Let $k_{0}=\arg \max _{j}\left(\max _{\nu / \nu_{0} \in \mathbb{B}} \widehat{u}_{j}^{\mathrm{NL}}(2 \pi \nu)\right)$ for which 
$\widehat{u}_{j}^{\mathrm{NL}}(2 \pi \nu)$ is the Fourier transform of $u_{j}^{\mathrm{NL}}(t)$. In the frequency domain, the observation $w(2 \pi \nu)$ corresponding to the selected blade out-plane displacement is defined by $w(2 \pi \nu)=\widehat{u}_{k_{0}}(2 \pi \nu)$. Figure 2 displays the graphs $\nu / \nu_{0} \mapsto w^{\mathrm{L}}(2 \pi \nu)$ (upper graph) and $\nu / \nu_{0} \mapsto w^{\mathrm{NL}}(2 \pi \nu)$ (lower graph) corresponding to a load intensity $f_{0}=2.75 \mathrm{~N}$. The spreading of the vibrational energy over the whole frequency band of analysis $\mathbb{B}$ is due to the nonlinear geometric effects and is characterized through secondary response peaks. For high frequencies that are located outside $\mathbb{B}_{e}^{2}$ in dimensionless frequency band $[3,3.34]$, the dynamical response induced by the geometric nonlinearities is negligible.

Nevertheless, some new resonances appear with the same order of magnitude than the main resonance in the dimensionless frequency band $\mathbb{B}_{s u b}=[1,1.5]$ (Note that, in this band, there exist several tuned eigenfrequencies of the structure, which are only excited through the chosen excitation under the linear assumption). It can be seen that the main resonance amplitude, located in $\mathbb{B}_{\text {sub }}$, is nearly twice the resonance amplitude located in $\mathbb{B}_{e}^{2}$. We put then in evidence a complex dynamical behavior that can be potentially dangerous because non-expected resonances with non-negligible amplitudes appear outside excitation frequency band $\mathbb{B}_{e}^{2}$.
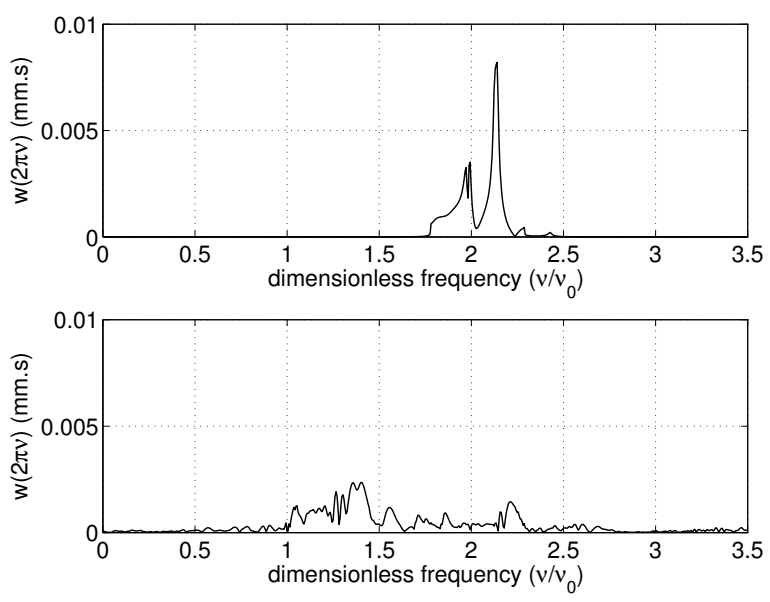

Figure 2: Frequency domain observation $\nu / \nu_{0} \mapsto w(2 \pi \nu)$ related to the linear (upper graph) and the nonlinear (lower graph) cases for $\mathbb{B}_{e}^{2}=[1.78,2.34]$ and $f_{0}=2.75 \mathrm{~N}$.

In the present case, the MEAN-NL-ROM is constructed by modal analysis without substructuring techniques. The uncertainties are not considered as independent from one blade to another one, which is coherent with the structure under consideration belonging to the class of integrated bladed disks, that are manufactured from a unique solid piece of metal. In the present analysis, for a better understanding of the phenomenon, only the matrices related to the linear part are random. The mistuning level is thus controlled by the $\mathbb{R}^{5}$-vector $\delta=\left(\delta_{M}, \delta_{D}, \delta_{C}, \delta_{K_{c}}, \delta_{K}\right)$. The analysis is focussed for the excitation frequency band $\mathbb{B}_{e}^{2}$ that exhibits the complex dynamic situation described above. The load intensity is fixed to $f_{0}=2.5 \mathrm{~N}$ and the uncertainty level is set to $\delta=\left(\delta_{M}, \delta_{D}, \delta_{C}, \delta_{K_{c}}, \delta_{K}\right)=(\delta, 0.2,0.2,0.2, \delta)$, in which $\delta=\delta_{M}=\delta_{K}$. Thus, the effects of mass and elastic uncertainties combined to uncertainties for the rotational effects are taken into account in the analysis. For fixed $\nu / \nu_{0} \in \mathbb{B}$, let $Y(2 \pi \nu)$ be the random dynamic amplification factor defined by

$$
Y(2 \pi \nu)=\frac{W(2 \pi \nu)}{\max _{\nu / \nu_{0} \in \mathbb{B}} w(2 \pi \nu)} .
$$




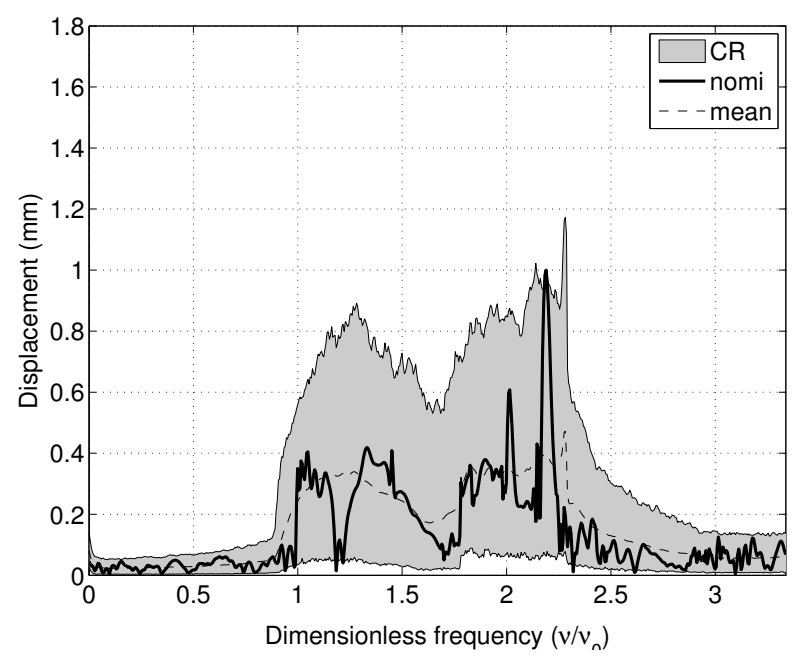

Figure 3: Stochastic analysis: frequency domain observation $Y^{\mathrm{NL}}(2 \pi \nu)$ related to the nonlinear case for $\delta_{K}=$ $\delta_{M}=0.16$ and for $\delta_{K_{c}}=\delta_{C}=\delta_{D}=0.2$ : mean model (thick line), mean of the stochastic model (thin dashed line), confidence region (gray region).
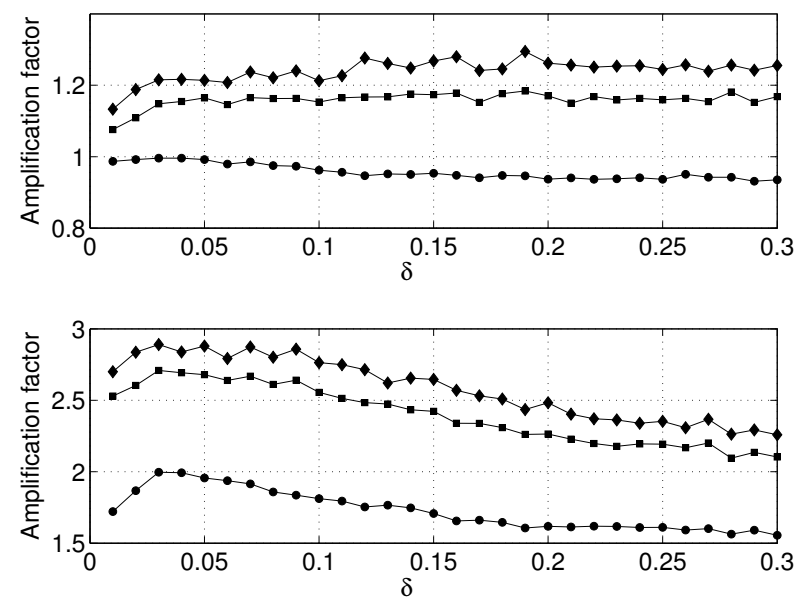

Figure 4: Quantile analysis of amplification factors $Y_{\infty}^{\mathrm{NL}}$ (upper graph) and $Z_{\infty}^{\mathrm{NL}}$ (lower graph) with respect to mistuning level $\delta$ with $P_{c}=0.5(\bullet$ symbol $), P_{c}=0.9(\boldsymbol{\text { symbol}}), P_{c}=0.95(\diamond$ symbol $)$

Figures 3 shows the confidence region of the nonlinear observation $Y^{\mathrm{NL}}(2 \pi \nu)$ for $\delta=0.16$. It is seen that the extreme values related to $Y^{\mathrm{NL}}(2 \pi \nu)$ yield moderate amplification even if the confidence region remains relatively broad. Although not presented in the present paper, it can be shown that, on the contrary, the linearized assumption clearly increases this amplification. It can then be deduced that the geometric nonlinear effects clearly inhibit the amplification of the random response in $\mathbb{B}_{e}^{2}$.

From now on, the analysis is focussed on the quantification of the amplification with respect to $\delta$. Let $Y_{\infty}$ be the random amplification factor defined by $Y_{\infty}=\max _{\nu / \nu_{0} \in \mathbb{B}} Y(2 \pi \nu)$. We then define the second random amplification factor, $Z_{\infty}$, such that

$$
Z_{\infty}=\frac{\max _{\nu / \nu_{0} \in \mathbb{B}_{\text {sub }}} W(2 \pi \nu)}{\max _{\nu / \nu_{0} \in \mathbb{B}_{\text {sub }}} w(2 \pi \nu)}
$$


Figure 4 compares the similar graphs obtained with random observations $Y_{\infty}^{\mathrm{NL}}$ and $Z_{\infty}^{\mathrm{NL}}$. Again, it is observed that the geometric nonlinear effects yield a limited sensitivity to mistuning uncertainties for observation $Y_{\infty}^{\mathrm{NL}}$. In Fig. 4, a special attention must be given to $Z_{\infty}^{\mathrm{NL}}$. In particular, these graphs exhibit a maximum, yielding the possibility to define some robustness areas that limit the dynamic amplification. It then points out, not only a complex sensitivity to uncertainties, but also high amplification levels that may yield unexpected amplifications.

\section{CONCLUSIONS}

The paper has presented an advanced methodology adapted to the mistuning analysis of bladed disks in the context of high amplitude loads inducing strong geometric nonlinear effects. The numerical results presented display new complex dynamical behaviors of the dynamical response of the blades. In particular, the numerical results demonstrate that the life duration of the industrial bladed disk can be very sensitive to the presence of geometric nonlinearities combined with mistuning effects.

\section{ACKNOWLEDGEMENTS}

This work was supported by the DGA (French defence procurement agency) in the context of the TURBODYNA project (project number ANR-13-ASTR-0008-01) related to the ANR ASTRID research program (specific support scheme for research works and innovation defence). SAFRAN Turbomeca is also acknowledged for giving permission to publish this work.

\section{REFERENCES}

[1] S.-T. Wei and C. Pierre, Localization Phenomena in Mistuned Assemblies with Cyclic Symmetry Part II: Forced Vibrations, ASME Journal of Vibration, Acoustics Stress and Reliability in Design, Vol. 110(4), (1988) pp. 439-449.

[2] E. Capiez-Lernout, C. Soize, Nonparametric modeling of random uncertainties for dynamic response of mistuned bladed disks, ASME Journal of Engineering Gas, Turbine and Power, Vol.126(3), (2004), pp. 610-618.

[3] M. Mbaye, C. Soize, J. Ousty, A reduced-order model of detuned cyclic dynamical systems with geometric modifications using a basis of cyclic modes, ASME Journal of Engineering for Gas Turbines and Power, Vol.132(11), (2010), paper 112502.

[4] M. Mbaye, C. Soize, J. Ousty, E. Capiez-Lernout, Robust Analysis of Design in Vibration of Turbomachines, ASME Journal of Turbomachinery, Vol.135(2), (2013), paper 021008.

[5] A.F. Vakakis, Dynamics of a nonlinear periodic structure with cyclic symmetry, Acta Mechanica, Vol.95(1-4), (1992), pp. 197-226.

[6] A. Grolet, F. Thouverez, Vibration analysis of a nonlinear system with cyclic symmetry, ASME Journal of Engineering for Gas Turbines and Power, Vol.133(2), (2011), paper 022502 .

[7] E. Capiez-Lernout, C. Soize, M.-P. Mignolet, Post-buckling nonlinear static and dynamical analyses of uncertain cylindrical shells and experimental validation Computer Methods in Applied Mechanics and Engineering, Vol. 271, (2014), pp. 210-230. 
[8] M.-P. Mignolet, A. Przekop, S.A. Rizzi, M.S. Spottswood, A review of indirect/nonintrusive reduced-order modeling of nonlinear geometric structures, Journal of Sound and Vibration, Vol.332(10), (2013), pp. 2437-2460.

[9] C. Soize, Stochastic Models of Uncertainties in Computational Mechanics, Lecture Notes in Engineering Mechanics 2, American Society of Civil Engineers (ASCE) (2012).

[10] C. Soize, Random matrix theory for modeling uncertainties in computational mechanics, Computer Methods in Applied Mechanics and Engineering, Vol. 194(12-16), (2005), pp. 1333-1366.

[11] E. Capiez-Lernout, C. Soize, M.-P. Mignolet, Computational stochastic statics of an uncertain curved structure with geometrical nonlinearity in three-dimensional elasticity Computational Mechanics, Vol. 49(1), (2012), pp. 87-97.

[12] M.-P. Mignolet, C. Soize, Stochastic reduced-order models for uncertain geometrically nonlinear dynamical systems, Computer Methods in Applied Mechanics and Engineering, Vol. 197, (2008), pp. 3951-3963.

[13] D.L. Thomas, Dynamics of rotationnally periodic structures, International Journal for Numerical Methods in Engineering, Vol. 14, (1979), pp. 81-102.

[14] A. Bossavit, Boundary value problems with symmetry and their approximation by finite elements, SIAM Journal of Applied Mathematics, Vol. 53, No. 5, (1993), pp. 1352-1380. 